\title{
Enlarged perivascular spaces and hemorrhagic transformation after acute ischemic stroke
}

\author{
Quhong Song ${ }^{1,2}$, Yajun Cheng ${ }^{1}$, Yanan Wang ${ }^{1}, J^{\prime}$ unfeng Liu ${ }^{1}$, Chenchen Wei ${ }^{3}$, Ming Liu ${ }^{1}$ \\ ${ }^{1}$ Department of Neurology, West China Hospital, Sichuan University, Chengdu, China; ${ }^{2}$ Department of Geriatrics and National Clinical Research \\ Center for Geriatrics, West China Hospital, Sichuan University, Chengdu, China; ${ }^{3}$ Department of Neurology, The Affiliated Hospital of Qingdao \\ University, Qingdao, China \\ Contributions: (I) Conception and design: Q Song, J Liu, M Liu; (II) Administrative support: M Liu; (III) Provision of study materials or patients: M \\ Liu; (IV) Collection and assembly of data: Q Song, Y Cheng, Y Wang, C Wei; (V) Data analysis and interpretation: Q Song, Y Cheng, Y Wang, J \\ Liu; (VI) Manuscript writing: All authors; (VII) Final approval of manuscript: All authors. \\ Correspondence to: Professor Ming Liu. Center of Cerebrovascular Disease, Department of Neurology, West China Hospital, Sichuan University, No. \\ 37, Guo Xue Xiang, Chengdu, China. Email: wyplmh@hotmail.com.
}

Background: Enlarged perivascular spaces (EPVS) are considered to be neuroimaging markers of cerebral small vessel disease (CSVD). It remains unknown whether EPVS are associated with hemorrhagic transformation (HT) after acute ischemic stroke (AIS). We performed this retrospective cohort study to explore the associations of EPVS with clinical risk factors and other CSVD imaging features, and to investigate the relationship between EPVS and HT in patients with AIS.

Methods: AIS patients admitted within 24 hours of stroke onset between January 2016 and December 2017 were consecutively enrolled. EPVS, lacunes, and white matter hyperintensities (WMH) were rated with validated rating scales on magnetic resonance images after the stroke. HT was defined as hemorrhage determined by follow-up brain imaging during the patients' hospital stay. Logistic regression was used to determine the risk factors and associations with other CSVD markers of EPVS in the basal ganglia (BG) and centrum semiovale (CS) regions, and the impact of EPVS on HT was further explored.

Results: Among 494 included patients (mean age 66.4 years, 58.1\% male), 81 (16.4\%) experienced HT. In the multivariate logistic analyses, increasing age [odd ratio (OR) 1.041, 95\% confidence interval (CI), 1.0171.066], hypertension (OR 2.174, 95\% CI, 1.338-3.532), lacunar stroke (OR 1.968, 95\% CI, 1.169-3.314), CS-EPVS (OR 2.474, 95\% CI, 1.796-3.407), and periventricular WMH (OR 2.140, 95\% CI, 1.441-3.176) were significantly associated with BG-EPVS; whereas only BG-EPVS (OR 4.349, 95\% CI, 2.281-8.291) were independently related to CS-EPVS. After adjustment for potential variables, neither BG-EPVS (OR 0.674, 95\% CI, 0.336-1.350) nor CS-EPVS (OR 0.792, 95\% CI, 0.334-1.879) was significantly associated with the occurrence of HT.

Conclusions: Our data showed that EPVS in the BG and CS regions were interrelated and had different risk factors in ischemic stroke patients. EPVS (particularly that in BG) were independently related to other CSVD markers. The presence or burden of EPVS was not significantly associated with HT after AIS.

Keywords: Enlarged perivascular spaces (EPVS); cerebral small vessel disease, hemorrhagic transformation; acute ischemic stroke (AIS)

Submitted Mar 29, 2021. Accepted for publication Jun 10, 2021.

doi: $10.21037 /$ atm-21-1276

View this article at: https://dx.doi.org/10.21037/atm-21-1276 


\section{Introduction}

Cerebral small vessel disease (CSVD) is a common agerelated pathological process of the microvasculature caused by various risk factors (1). Classic neuroimaging features of CSVD include lacunes, white matter hyperintensities (WMH), and cerebral microbleeds (CMB) $(1,2)$. Recently, enlarged perivascular spaces (EPVS) have been increasingly reported as an emerging marker of CSVD (3). Perivascular spaces (PVS), also known as Virchow-Robin spaces, are fluid-filled compartments surrounding the penetrating vessels, which facilitate interstitial fluid drainage and the removal of metabolites from the brain (4). Although normally undetectable, they may enlarge and become apparent with advancing age and increased exposure to vascular risk factors $(4,5)$. When enlarged, PVS can be detected on T2-weighted magnetic resonance imaging (MRI) as punctate or linear hyperintensities, particularly in the basal ganglia (BG) and centrum semiovale $(2,6)$.

Initial pathologic studies found that EPVS seldom cause damage in the brain parenchyma, and hence were regarded as normal variants with limited clinical importance (7). Recently, some studies in aging adults demonstrated that EPVS were associated with an increased risk of incident stroke, dementia, and depression (8-10). Moreover, growing evidence indicates that EPVS are more prevalent in stroke patients $(11,12)$, and a high EPVS burden can predict poor outcomes after stroke $(11,13,14)$. However, few studies have explored the relationship of EPVS to hemorrhagic transformation (HT) following ischemic stroke. Whether EPVS play a role in HT remains uncertain. EPVS are reported to correlate with other CSVD markers (i.e., WMH and CMB) $(2,3,5)$, and previous studies have shown that WMH and CMB may increase the risk of HT (15-17). EPVS were also found to be associated with blood brain barrier dysfunction $(1,4)$, which is considered to be the basic pathophysiology of HT (18). We therefore hypothesized that EPVS may be related to the occurrence of HT.

In the present study, we investigated the cross-sectional associations of EPVS with clinical factors and other CSVD markers, and further identified the relationship between EPVS and HT in patients with acute ischemic stroke (AIS).

We present the following article in accordance with the STROBE reporting checklist (available at https://dx.doi. org/10.21037/atm-21-1276).

\section{Methods}

\section{Study population}

This retrospective cohort study was derived from the Chengdu Stroke Registry, which has prospectively and consecutively recruited ischemic stroke patients admitted to Department of Neurology in West China Hospital since 2002. The details of the registry have been described previously (19). In the present study, we enrolled adult patients with ischemic stroke admitted within 24 hours after stroke onset from January 2016 to December 2017. Ischemic stroke was diagnosed according to the World Health Organization criteria (20), and confirmed by computed tomography (CT) or MRI scanning. Patients were excluded if they did not undergo MRI scans due to contraindications (e.g., claustrophobia, implanted devices incompatible with MRI) or had a severe stroke and were unable to bear MRI scanning; or if the MRI image quality was poor, making the assessment of EPVS difficult. This study was approved by the Biomedical Research Ethics Committee of West China Hospital, Sichuan University (No. 2016-339), and conformed to the provisions of the Declaration of Helsinki (as revised in 2013). Since the study was a retrospective cohort, individual consent was waived.

\section{Data collection}

Patients' baseline demographic and clinical data were retrieved as follows: age, sex, time from stroke onset to admission, medical history [hypertension, diabetes mellitus, dyslipidemia, atrial fibrillation (AF), previous stroke or transient ischemic attack (TIA)], history of smoking and alcohol consumption, National Institutes of Health Stroke Scale (NIHSS) score, blood pressure, laboratory data, the Trial of ORG 10172 in Acute Stroke Treatment (TOAST) classification, and treatments after admission (antiplatelet, anticoagulant, lipid lowering, and reperfusion therapy). The laboratory data included platelet count, neutrophilto-lymphocyte ratio (NLR), blood glucose, creatinine, uric acid, and coagulation testing. All venous blood samples were obtained within 24 hours after admission. 


\section{Image acquisition}

Brain CT was performed within 24 hours after admission, followed by regular MRI scanning within 7 days of admission or CT immediately in case of clinical worsening. CT was performed using a 64-section scanner with 7-mm slice thickness (Siemens). MRI was performed on a 3.0 T scanner with 5-mm slice thickness and matrix size was 256×256 pixels (Magnetom, Siemens, Erlangen, Germany), including the following sequences: T1-weighted, T2weighted, and fluid-attenuated inversion recovery (FLAIR) imaging. The parameters of the above MRI sequences have been described detailly in our previous study (17).

\section{Rating of EPVS}

MRI findings were categorized and recorded in accordance with STRIVE (Standards for Reporting Vascular Changes on Neuroimaging) criteria (2). All images were assessed by two experienced neurologists (QS and YC) blinded to patients' clinical information. In case of divergence, a third neurologist (JL) was consulted and a consensus was reached.

EPVS were defined as fluid-filled spaces surrounding the penetrating vessels, with a signal intensity identical to cerebrospinal fluid (CSF) on all MRI sequences. Their appearance was linear, round or ovoid with a diameter generally smaller than $3 \mathrm{~mm}$ and without the surrounding FLAIR hyperintense rim (2). EPVS were counted in the $\mathrm{BG}$ and centrum semiovale (CS) using the following 5-point rating scale: $0=$ none EPVS, $1=1$ to $10,2=11$ to $20,3=21$ to 40 , and $4=>40$ EPVS $(3,6)$. For both regions, we chose the slice in the most affected hemisphere only. When the infarct size was too large to count the EPVS, only the contralateral hemisphere was rated. Moderate-to-severe EPVS were defined as having a score $\geq 2$ in the BG or CS. Total EPVS burden was calculated by summing the BG and CS-EPVS scores. In this study, the inter-rater kappa values were 0.75 and 0.61 for BG and CS-EPVS, respectively.

\section{Other CSVD markers}

Lacunes were defined as round or ovoid lesions with a diameter of $3-15 \mathrm{~mm}$, located in the $\mathrm{BG}$, internal or external capsule, thalamus, centrum semiovale, or brainstem, showing CSF-like intensities on T2-weighted and FLAIR images, generally with a hyperintense rim on FLAIR (2). The number of lacunes was identified. WMH was rated according to the Fazekas scale, and divided into periventricular white matter hyperintensity $(\mathrm{PWMH})$ and deep white matter hyperintensity (DWMH) (21). PWMH was graded as absent (score 0 ), cap (score 1), smooth halo (score 2), or irregular and extending into the subcortical white matter (score 3), while DWMH was rated as absent (score 0 ), punctate foci (score 1), early confluent (score 2), or confluent (score 3). Extensive WMH was defined as PWMH score $=3$ or DWMH score $\geq 2$. The inter-rater kappa values were 0.65 for lacunes, 0.93 for PWMH, 0.90 for DWMH, respectively.

Several CSVD markers often coexist in a patient, and it seems better to quantify the total burden of CSVD to assess the cumulative effect of small vessel injury on the whole brain. Thus, in this study, we used lacunes, WMH and EPVS to calculate the "total CSVD burden" (ranging from 0 to 3 ) based on the validated ordinal scale $(22,23)$ : One point was awarded if lacunes $\geq 1$, or PVWMH score $=3$ and/ or DWMH score $\geq 2$, or BG-EPVS score $\geq 2$.

\section{Outcome assessment}

The main outcome measure was development of hemorrhagic transformation (HT) during patients' stay in the hospital. HT was defined as either hemorrhage within the infarcted area or parenchyma hemorrhage outside the infarct zone, which was observed on follow-up CT or MRI during hospitalization but not on the initial brain CT (24). Two trained neurologists (YW and CW) without knowledge of patient's clinical information independently assessed the presence of HT. Disagreements were solved through discussion or advice from a third neurologist (ML). HT was further classified as hemorrhagic infarction (HI) or parenchymal hematoma (PH) based on the European Cooperative Acute Stroke Study (ECASS) III criteria (25). Symptomatic HT (sHT) was defined as any intracranial hemorrhage associated with any decline in neurologic status according to National Institute of Neurological Diseases and Stroke (NINDS) criteria (26).

\section{Statistical analysis}

In this study, values were missing for platelet count in two $(0.4 \%)$ patients. Data for all other variables were complete. As the level of missing data was low $(<5 \%)$ and almost negligible, we decided not to impute the missing data.

Continuous data were expressed as mean with standard deviation (SD) or median with interquartile range (IQR), while categorical data were reported as numbers and 
percentages. We firstly assessed the correlation between EPVS and other CSVD features (the number of lacunes and the ordinal scores of WMH) using Spearman's rank correlation. Then, the univariate and multivariate logistic analyses were performed to study the association of EPVS with clinical risk factors and CSVD markers. As EPVS scores were not normally distributed, we dichotomized EPVS into mild total EPVS (score 0-2) vs. moderate-tosevere total EPVS [3-8], mild BG-EPVS [0-1] vs. moderateto-severe BG-EPVS [2-4], and mild CS-EPVS [0-1] vs. moderate-to-severe CS-EPVS [2-4], respectively. Potential variables identified from the recent literature $(11,12,27)$ were adjusted: age, sex, hypertension, diabetes mellitus, dyslipidemia, AF, prior stroke/TIA, smoking, drinking, lacunar stroke, PWMH, DWMH, and lacunes.

For our second aim, we compared clinical and imaging characteristics of patients with HT to those without HT. Intergroup differences in categorical variables were assessed using $\chi^{2}$ test or Fisher's exact test, while differences in continuous variables were compared using Student's $t$-test or Mann-Whitney $\mathrm{U}$ test, as appropriate. Then, multivariate logistic analyses were used to investigate the independent association between EPVS and HT, incorporating variables with $\mathrm{P}<0.1$ identified in univariate analysis.

All statistical analyses were performed using SPSS 26.0 (IBM, USA), and a 2 -sided $\mathrm{P}<0.05$ was considered to be statistically significant.

\section{Results}

\section{Baseline characteristics}

During the study period, 627 patients within 24 hours of stroke onset were admitted to our hospital, and 133 patients were excluded for the following reasons: no MRI scans $(\mathrm{n}=108)$, and poor quality of MRI images $(\mathrm{n}=25)$. Therefore, 494 patients were finally included in this study (Figure S1). The mean age of the study population was $66.4 \pm 13.4$ years (58.1\% male), and the median NIHSS score was 5 (IQR, $2-11)$. The baseline characteristics of included patients were presented in Table 1. A total of 57 (11.5\%) patients received reperfusion therapy after admission: $41(8.3 \%)$ patients with thrombolytic therapy, 13 (2.6\%) with endovascular therapy, and $3(0.6 \%)$ with thrombolysis followed by endovascular therapy.

The median time from stroke onset to MRI scanning was 2.7 days (IQR, 1.8-3.7 days). EPVS were detected in 99.8\% (493/494) patients, and the median EPVS scores in $B G$ and $C S$ were 1 (IQR, 1-2) and 2 (IQR, 2-3), respectively. Overall, 48.4\% (239/494) of patients were rated as moderate-to-severe BG-EPVS, 88.1\% (435/494) as moderate-to-severe CS-EPVS, and 46.6\% (230/494) had both moderate-to-severe BG-EPVS and moderate-tosevere CS-EPVS.

\section{EPVS and other CSVD markers}

Compared to CS-EPVS, BG-EPVS were more closely related to other imaging markers of CSVD, with the strongest correlation for PWMH $(\rho=0.515, \mathrm{P}<0.001)$, followed by DWMH $(\rho=0.450, \mathrm{P}<0.001)$ and lacunes $(\rho=0.237, P<0.001)$ (Table S1). There was a correlation between BG and CS-EPVS $(\rho=0.243, \mathrm{P}<0.001)$, but no significant correlation was found among CS-EPVS, lacunes and WMH (all $\mathrm{P}>0.05$ ) (Table S1). The multivariate logistic analyses showed that DWMH was independently associated with total EPVS (OR 2.012, 95\% CI, 1.062-3.809) (Table 2); age (OR 1.041, 95\% CI, 1.017-1.066), hypertension (OR 2.174, 95\% CI, 1.338-3.532), lacunar stroke (OR 1.968, 95\% CI, 1.169-3.314), CS-EPVS (OR 2.474, 95\% CI, 1.796-3.407), and PWMH (OR 2.140, 95\% CI, 1.4413.176) were significantly associated with EPVS in BG; whereas only BG-EPVS (OR 4.349, 95\% CI, 2.281-8.291) were independently related to CS-EPVS (Table 2).

\section{EPVS and HT}

The median length of hospital stay was 11 days (IQR, $8-16$ days), and there were 81 (16.4\%) patients experienced HT during hospitalization (median time from onset to HT 3.5 days, IQR, 2-6 days), of whom 43 (8.7\%) with HI, 38 (7.7\%) with $\mathrm{PH}$, and 10 (2.0\%) with sHT.

The baseline clinical and imaging characteristics of patients with HT and without HT were shown in Table 1. Patients with HT were older, more likely to be female, had lower onset to admission time, higher proportion of AF and reperfusion therapy, higher NIHSS score, lower systolic pressure, lower proportion of alcohol consumption and antiplatelet therapy than those without HT (all $\mathrm{P}<0.05$ ), while the application of anticoagulants was similar among the two groups $(17.3 \%$ vs. $10.7 \%, \mathrm{P}=0.09)$. With respect to laboratory data, compared to those without HT, patients experienced HT had lower platelet count and higher NLR level (all $\mathrm{P}<0.05$ ) (Table 1).

As for CSVD markers, both the median BG-EPVS score and the proportion of moderate-to-severe BG-EPVS 
Table 1 Baseline characteristics of patients with and without HT

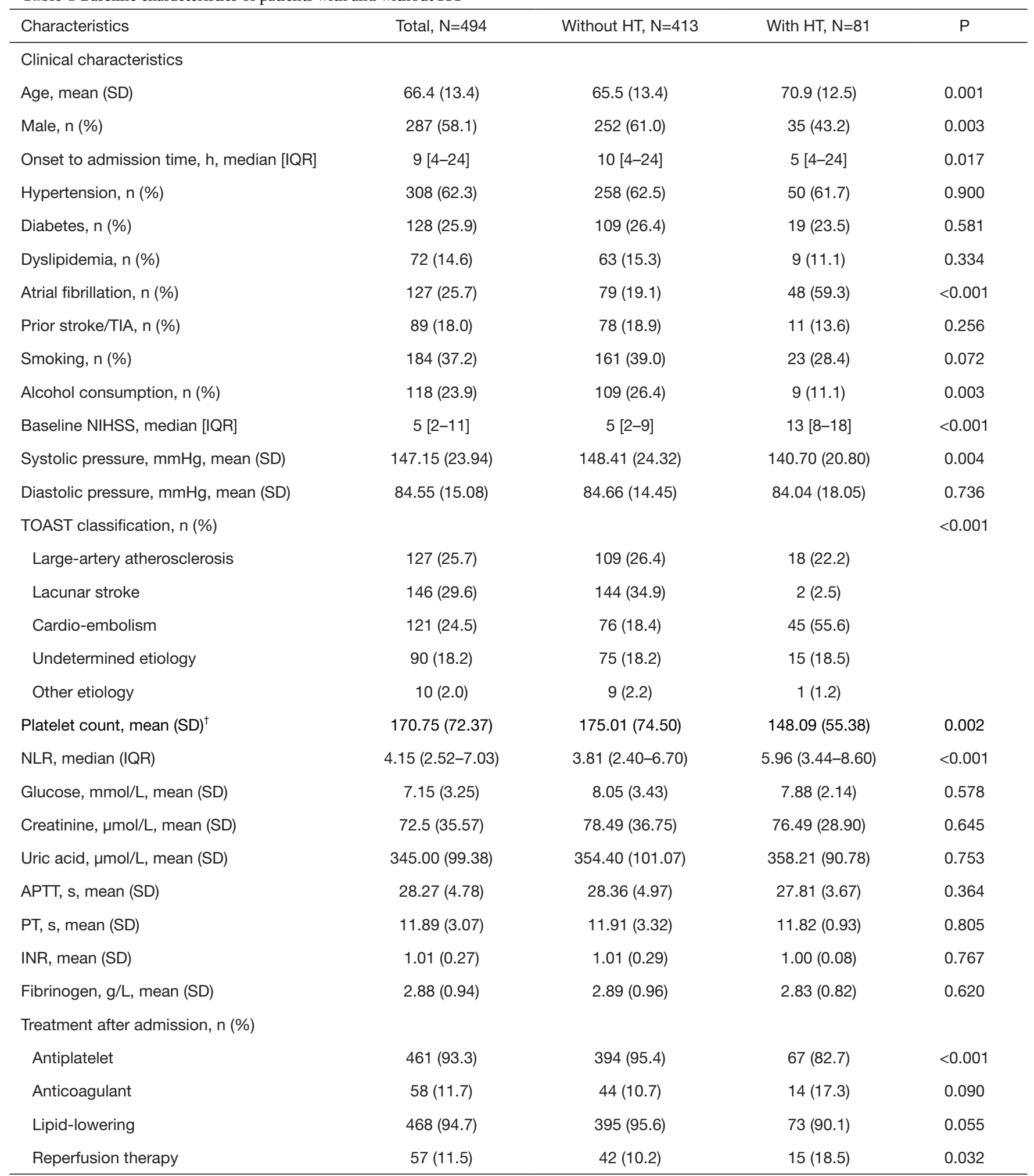

Table 1 (continued) 
Table 1 (continued)

\begin{tabular}{|c|c|c|c|c|}
\hline Characteristics & Total, $\mathrm{N}=494$ & Without $\mathrm{HT}, \mathrm{N}=413$ & With $\mathrm{HT}, \mathrm{N}=81$ & $\mathrm{P}$ \\
\hline Infarct area >1/3MCA, $n(\%)$ & $141(28.5)$ & $81(19.6)$ & $60(74.1)$ & $<0.001$ \\
\hline Total EPVS, median [IQR] & $4[3-5]$ & $4[3-5]$ & $4[3-4]$ & 0.001 \\
\hline BG-EPVS, median [IQR] & $1[1-2]$ & $2[1-2]$ & $1[1-2]$ & 0.005 \\
\hline CS-EPVS, median [IQR] & $2[2-3]$ & $2[2-3]$ & $2[2-3]$ & 0.019 \\
\hline Moderate to severe CS-EPVS, n (\%) & $435(88.1)$ & $368(89.1)$ & $67(82.7)$ & 0.105 \\
\hline Lacunes, n (\%) & $95(19.2)$ & $85(20.6)$ & $10(12.3)$ & 0.086 \\
\hline PWMH, median [IQR] & $1[0-2]$ & $1[0-2]$ & $1[0-1]$ & 0.195 \\
\hline Extensive DWMH, n (\%) & $90(18.2)$ & $78(18.9)$ & $12(14.8)$ & 0.385 \\
\hline cWMH, median [IQR] & $2[0-3]$ & $2[1-3]$ & $1[0-2]$ & 0.141 \\
\hline No cWMH (0), n (\%) & $128(25.9)$ & $103(24.9)$ & $25(30.9)$ & 0.287 \\
\hline Mild cWMH (1-2), n (\%) & $228(46.2)$ & $188(45.5)$ & $40(49.4)$ & \\
\hline Moderate cWMH (3-4), n (\%) & $96(19.4)$ & $86(20.8)$ & $10(12.3)$ & \\
\hline Severe cWMH (5-6), n (\%) & $42(8.5)$ & $36(8.7)$ & $6(7.4)$ & \\
\hline Total CSVD burden, median [IQR] & $1[0-1]$ & $1[0-2]$ & $0[0-1]$ & 0.003 \\
\hline Score 0, n (\%) & $219(44.3)$ & $171(41.4)$ & $48(59.3)$ & 0.025 \\
\hline
\end{tabular}

${ }^{\dagger}$ Platelet count was missing in $2(0.4 \%)$ patients. HT, hemorrhagic transformation; TIA, transient ischemic attack; NIHSS, National Institutes of Health Stroke Scale; TOAST, Trial of ORG 10,172 in Acute Stroke Treatment; NLR, neutrophil to lymphocyte ratio; APTT, activated partial thromboplastic time; PT, prothrombin time; INR, international normalized ratio; MCA, middle cerebral artery; BG-EPVS, basal ganglia enlarged perivascular spaces; CS-EPVS, centrum semiovale enlarged perivascular spaces; $\mathrm{PWMH}$, periventricular white matter hyperintensity; DWMH, deep white matter hyperintensity; cWMH, total cerebral white matter hyperintensity; CSVD, cerebral small vessel disease; SD, standard deviation; IQR, interquartile range; reperfusion therapy refers to thrombosis and endovascular therapy.

were lower in patients with HT (all $\mathrm{P}<0.05$ ). No significant difference was observed in moderate-to-severe CS-EPVS, lacunes, and WMH between patients with vs. without HT (all $\mathrm{P}>0.05$ ) (Table 1). In the multivariate analyses, after adjusting for potential confounders, neither BG-EPVS nor CS-EPVS was significantly associated with HT (Table 3); whereas $\mathrm{AF}$ and infarct size $>1 / 3$ middle cerebral artery (MCA) were found to be the independent predictors of HT, which may increase HT risk by 2.5 -fold and 7-fold, respectively (all $\mathrm{P}<0.05$, data not shown).

In additional exploratory analysis, we investigated the relationship between "total CSVD burden" and HT. As shown in Table 3, after adjustment for covariates, no significant association of "total CSVD burden" with HT was found. We further explored the relationship between CSVD markers and the presence of $\mathrm{PH}$, and the results showed that there were no remarkable differences in CSVD features between $\mathrm{PH}$ and non-PH group (Table S2). 
Table 2 Univariate and multivariate associations of clinical risk factors and other CSVD markers with EPVS

\begin{tabular}{|c|c|c|c|c|c|c|}
\hline Variables & \multicolumn{2}{|c|}{ Total EPVS (0-2 vs. 3-8) } & \multicolumn{2}{|c|}{ BG-EPVS (0-1 vs. 2-4) } & \multicolumn{2}{|c|}{ CS-EPVS (0-1 vs. 2-4) } \\
\hline Male & $0.916(0.505-1.663)$ & $1.112(0.500-2.473)$ & $1.074(0.751-1.536)$ & $1.274(0.711-2.282)$ & $1.022(0.589-1.772)$ & $1.071(0.512-2.240)$ \\
\hline Dyslipidemia & $1.280(0.525-3.125)$ & $1.365(0.539-3.456)$ & $0.831(0.503-1.373)$ & $0.834(0.448-1.553)$ & $1.100(0.499-2.426)$ & $1.250(0.523-2.988)$ \\
\hline AF & 1.107 (0.559-2.191) & $0.974(0.441-2.150)$ & $0.667(0.444-1.004)$ & $0.570(0.324-1.289)$ & $1.128(0.597-2.133)$ & $1.204(0.560-2.589)$ \\
\hline $\begin{array}{l}\text { Prior } \\
\text { stroke/TIA }\end{array}$ & $1.686(0.695-4.088)$ & $1.393(0.521-3.723)$ & $1.733(1.087-2.763)$ & $0.703(0.383-1.289)$ & 1.459 (0.666-3.193) & $1.377(0.575-3.298)$ \\
\hline $\begin{array}{l}\text { Lacunar } \\
\text { stroke }\end{array}$ & $1.088(0.568-2.084)$ & $0.830(0.395-1.746)$ & 2.639 (1.766-3.943) & $1.968(1.169-3.314)$ & $0.950(0.526-1.715)$ & $0.662(0.330-1.328)$ \\
\hline BG-EPVS & - & - & - & - & $3.822(2.160-6.763)$ & 4.349 (2.281-8.291) \\
\hline CS-EPVS & - & - & 2.056 (1.589-2.660) & $2.474(1.796-3.407)$ & - & - \\
\hline PWMH & $1.937(1.311-2.862)$ & $1.218(0.676-2.194)$ & $3.430(2.654-4.434)$ & $2.140(1.441-3.176)$ & $1.344(0.980-1.844)$ & $0.677(0.395-1.162)$ \\
\hline DWMH & $2.385(1.483-3.834)$ & $2.012(1.062-3.809)$ & $3.140(2.389-4.127)$ & $1.363(0.928-2.001)$ & $1.565(1.076-2.277)$ & $1.495(0.866-2.579)$ \\
\hline Lacunes & $1.094(0.512-2.337)$ & 0.497 (0.204-1.209) & $2.973(1.841-4.802)$ & $1.377(0.732-2.590)$ & $0.924(0.469-1.818)$ & $0.536(0.233-1.233)$ \\
\hline
\end{tabular}

The multivariate analyses adjusted for all covariates presented in the table. AF, atrial fibrillation; TIA, transient ischemic attack; CSVD, cerebral small vessel disease; BG-EPVS, basal ganglia enlarged perivascular spaces; CS-EPVS, centrum semiovale enlarged perivascular spaces; $\mathrm{PWMH}$, periventricular white matter hyperintensity; $\mathrm{DWMH}$, deep white matter hyperintensity; OR, odds ratio; $\mathrm{Cl}$, confidence interval.

\section{Discussion}

In the present study, we found that EPVS were common in patients with ischemic stroke, and EPVS in CS were more predominant than that in BG. After adjusting for potential covariates, total EPVS were independently associated with DWMH; CS-EPVS and BG-EPVS were interrelated; and BG-EPVS were significantly related to increasing age, hypertension, lacunar stroke, and PWMH. Regarding to their role in HT, the multivariate analyses showed that neither BG-EPVS nor CS-EPVS had an independent association with the occurrence of HT after ischemic stroke.

Our findings concerning the relationship of BGEPVS with age, hypertension and WMH were consistent across different populations including lacunar stroke (28), intracerebral hemorrhage (12), and cognitive impairment (29). Besides, the stronger association between lacunar stroke and EPVS in BG than that in CS was similar to previous studies $(3,30,31)$. However, none of any interested clinical-radiological factors (except for BG-EPVS) was identified to be independently associated with CS-EPVS. Some studies have indicated that the spatial distributions of EPVS may reflect distinct small arteriopathies such that BG-EPVS is likely caused by hypertensive arteriopathy $(5,32)$, whereas CS-EPVS may be driven by cerebral amyloid angiopathy $(4,32,33)$. The significant but weak correlation between BG and CS-EPVS supports such different pathophysiological mechanisms contributing to each EPVS distribution. Our results add to evidence on the association of EPVS with CSVD markers, suggesting that EPVS (particularly that in BG) are imaging manifestation of CSVD rather than incidental findings.

Several CSVD markers have been reported to associate with an increased risk of HT (15-17). However, studies 
Table 3 Univariate and multivariate associations between EPVS and HT

\begin{tabular}{|c|c|c|c|c|c|c|c|c|}
\hline Imaging variables & \multicolumn{2}{|l|}{ Unadjusted } & \multicolumn{2}{|l|}{ Model 1} & \multicolumn{2}{|l|}{ Model 2} & \multicolumn{2}{|l|}{ Model 3} \\
\hline Total EPVS & $0.723(0.589-0.887)$ & 0.002 & $0.749(0.581-0.965)$ & 0.025 & $0.770(0.593-1.000)$ & 0.050 & $0.817(0.617-1.081)$ & 0.157 \\
\hline BG-EPVS & $0.638(0.454-0.897)$ & 0.010 & $0.681(0.448-1.037)$ & 0.073 & $0.703(0.459-1.078)$ & 0.106 & $0.785(0.499-1.235)$ & 0.294 \\
\hline $\begin{array}{l}\text { BG-EPVS } \\
(0-1 \text { vs. } 2-4)\end{array}$ & $0.474(0.287-0.782)$ & 0.003 & $0.571(0.304-1.076)$ & 0.083 & $0.595(0.313-1.130)$ & 0.113 & $0.674(0.336-1.350)$ & 0.265 \\
\hline $\begin{array}{l}\text { CS-EPVS } \\
(0-1 \text { vs. } 2-4)\end{array}$ & $0.585(0.304-1.125)$ & 0.108 & $0.530(0.242-1.160)$ & 0.112 & $0.583(0.262-1.300)$ & 0.188 & $0.792(0.334-1.879)$ & 0.597 \\
\hline Total CSVD burden & $0.669(0.500-0.895)$ & 0.007 & $0.721(0.495-1.051)$ & 0.089 & $0.725(0.495-1.062)$ & 0.099 & $0.813(0.533-1.240)$ & 0.337 \\
\hline Score 3 & $0.460(0.155-1.366)$ & 0.162 & $0.631(0.164-2.435)$ & 0.504 & $0.708(0.184-2.729)$ & 0.708 & $1.260(0.287-5.524)$ & 0.759 \\
\hline
\end{tabular}

Model 1: adjusted for age, sex, stroke onset to admission time, AF, smoking, alcohol consumption, baseline NIHSS, systolic pressure, TOAST classification, platelet, NLR; Model 2: model 1+ treatment after admission; Model 3: model 2+ Infarct area >1/3MCA. HT, hemorrhagic transformation; BG-EPVS, basal ganglia enlarged perivascular spaces; CS-EPVS, centrum semiovale enlarged perivascular spaces; CSVD, cerebral small vessel disease; OR, odds ratio; Cl, confidence interval.

focused on EPVS in the development of HT are rare. A previous study observed no significant relationship between EPVS and HT in ischemic stroke patients with AF and/ or rheumatic heart disease (34), which was in line with the results of our present study. Nevertheless, another recent study, performed on 1386 patients with AF and recent TIA or ischemic stroke, found that BG-EPVS were associated with spontaneous intracranial hemorrhage occurred during follow-up (mean follow-up period 2.34 years) (35). Unlike the above study concentrated mainly on anticoagulantrelated intracranial hemorrhage (median time from anticoagulation initiation to hemorrhage 272 days, IQR, 211-657 days) (35), we focused on acute hemorrhage following ischemic stroke (median time from stroke onset to hemorrhage 3.5 days, IQR, 2-6 days); in addition, variations in study population and ethnicities may be another explanation for the difference between our results and that of the above study.

CSVD features frequently occur together and focusing only on one or two individual CSVD markers while disregarding the others seems artificial. Recently, some researchers have proposed to calculate the total CSVD burden (22,23), which combines lacunes, WMH, CMB and EPVS to reflect the overall effect of small vessel injury. In this retrospective study, assessment of CMB was unavailable as susceptibility weighted imaging and gradient recalled echo sequences were not the routine MRI sequence for ischemic stroke patients in our hospital. Therefore, in our exploratory analysis, we only used lacunes, WMH and EPVS that can be available on routine MRI sequences to calculate the "total CSVD burden". Our results showed that the "total CSVD burden" was lower in patients with HT (Table 1). Further analysis found that the proportion of patients with infarct area $>1 / 3$ MCA decreased with the increasing of "total CSVD burden" (score 0 vs. 1 vs. 2 vs. $3: 37.0 \%$ vs. $25.5 \%$ vs. $19.3 \%$ vs. $11.4 \%, \mathrm{P}=0.001$ ). As the presence of CSVD may reflect the local chronic ischemia and hypoxia (36), we speculate that the coexistence of several CSVD markers may exert effects like those of ischemic preconditioning (37), improving the tolerance to subsequent ischemic injury and thus reducing the risk of HT. Furthermore, previous studies have found that the presence of other CSVD markers may weaken even reverse the effect of $\mathrm{CMB}$ on $\mathrm{HT}$, making $\mathrm{CMB}$ exert protective rather than harmful effects (34). This finding further supports the underlying neuroprotective effect of CSVD markers. However, in the multivariate analysis incorporating clinical factors, there was no significant 
relationship between "total CSVD burden" and HT, and only AF and infarct size $>1 / 3 \mathrm{MCA}$ were found to be independently related to HT. In this study, as lack of $\mathrm{CMB}$ data, the impact of total CSVD burden on HT might be underestimated. Future prospective multicenter studies with large samples are needed to clarify the actual association between total CSVD burden and HT.

Our study has several limitations. First, this study was a retrospective analysis based on a single center stroke registry, and our data may not represent the overall Chinese population or other ethnicities. Second, potential selection bias may be introduced. Patients with MRI contraindications, with severe stroke unable to undergo MRI, and with poor image quality were excluded, which may bias the association between EPVS and HT. Third, $\mathrm{CMB}$ was not assessed in the present study, thus the relationship between EPVS and CMB was not explored, and the association between total CSVD burden and HT may also be underestimated. Finally, data about HT locations (lobar vs. deep/infratentorial) were not collected, and whether EPVS were associated with different sites of HT was unknown and required further investigation.

\section{Conclusions}

Our study found that EPVS were common in patients with ischemic stroke, and EPVS in BG and CS region were interrelated and had different risk factors. EPVS (particularly that in BG) were significantly associated with other CSVD markers. The presence or burden of EPVS was not associated with the risk of $\mathrm{HT}$ after ischemic stroke. More prospective multicenter studies are needed to confirm our results, and to explore the underlying mechanisms and clinical importance of EPVS.

\section{Acknowledgments}

We would like to thank professor Marc Fisher (Department of Neurology, Beth Israel Deaconess Medical Center, Harvard Medical School, Boston, MA, USA) for his help in polishing our paper.

Funding: This work was supported by the Major International (Regional) Joint Research Project, National Natural Science Foundation of China (81620108009), the National Natural Science Foundation of China (81901199), and the 1.3.5 project for disciplines of excellence, West China Hospital, Sichuan University (ZYGD18009).

\section{Footnote}

Reporting Checklist: The authors have completed the STROBE reporting checklist. Available at https://dx.doi. org/10.21037/atm-21-1276

Data Sharing Statement: Available at https://dx.doi. org/10.21037/atm-21-1276

Conflicts of Interest: All authors have completed the ICMJE uniform disclosure form (available at https://dx.doi. org/10.21037/atm-21-1276). All authors report that this work was supported by the Major International (Regional) Joint Research Project, National Natural Science Foundation of China (81620108009), the National Natural Science Foundation of China (81901199), and the 1.3.5 project for disciplines of excellence, West China Hospital, Sichuan University (ZYGD18009).

Ethical Statement: The authors are accountable for all aspects of the work in ensuring that questions related to the accuracy or integrity of any part of the work are appropriately investigated and resolved. This study was approved by the Biomedical Research Ethics Committee of West China Hospital, Sichuan University (No. 2016339), and conformed to the provisions of the Declaration of Helsinki (as revised in 2013). Since the study was a retrospective cohort, individual consent was waived.

Open Access Statement: This is an Open Access article distributed in accordance with the Creative Commons Attribution-NonCommercial-NoDerivs 4.0 International License (CC BY-NC-ND 4.0), which permits the noncommercial replication and distribution of the article with the strict proviso that no changes or edits are made and the original work is properly cited (including links to both the formal publication through the relevant DOI and the license). See: https://creativecommons.org/licenses/by-nc-nd/4.0/.

\section{References}

1. Wardlaw JM, Smith C, Dichgans M. Small vessel disease: mechanisms and clinical implications. Lancet Neurol 2019;18:684-96.

2. Wardlaw JM, Smith EE, Biessels GJ, et al. Neuroimaging standards for research into small vessel disease and its contribution to ageing and neurodegeneration. Lancet 
Neurol 2013;12:822-38

3. Potter GM, Doubal FN, Jackson CA, et al. Enlarged perivascular spaces and cerebral small vessel disease. Int J Stroke 2015;10:376-81.

4. Brown R, Benveniste H, Black SE, et al. Understanding the role of the perivascular space in cerebral small vessel disease. Cardiovasc Res 2018;114:1462-73.

5. Yakushiji Y, Charidimou A, Hara M, et al. Topography and associations of perivascular spaces in healthy adults: the Kashima scan study. Neurology 2014;83:2116-23.

6. Potter GM, Chappell FM, Morris Z, et al. Cerebral perivascular spaces visible on magnetic resonance imaging: development of a qualitative rating scale and its observer reliability. Cerebrovasc Dis 2015;39:224-31.

7. Jungreis CA, Kanal E, Hirsch WL, et al. Normal perivascular spaces mimicking lacunar infarction: $M R$ imaging. Radiology 1988;169:101-4.

8. Debette S, Schilling S, Duperron MG, et al. Clinical Significance of Magnetic Resonance Imaging Markers of Vascular Brain Injury: A Systematic Review and Metaanalysis. JAMA Neurol 2019;76:81-94.

9. Ding J, Sigurethsson S, Jonsson PV, et al. Large Perivascular Spaces Visible on Magnetic Resonance Imaging, Cerebral Small Vessel Disease Progression, and Risk of Dementia: The Age, Gene/Environment Susceptibility-Reykjavik Study. JAMA Neurol 2017;74:1105-12.

10. van Sloten TT, Sigurdsson S, van Buchem MA, et al. Cerebral Small Vessel Disease and Association With Higher Incidence of Depressive Symptoms in a General Elderly Population: The AGES-Reykjavik Study. Am J Psychiatry 2015;172:570-8.

11. Lau KK, Li L, Lovelock CE, et al. Clinical Correlates, Ethnic Differences, and Prognostic Implications of Perivascular Spaces in Transient Ischemic Attack and Ischemic Stroke. Stroke 2017;48:1470-7.

12. Wang X, Feng H, Wang Y, et al. Enlarged Perivascular Spaces and Cerebral Small Vessel Disease in Spontaneous Intracerebral Hemorrhage Patients. Front Neurol 2019;10:881.

13. Liang Y, Deng M, Chen YK, et al. Enlarged perivascular spaces are associated with health-related quality of life in patients with acute ischemic stroke. CNS Neurosci Ther 2017;23:973-9.

14. Liang Y, Chan YL, Deng M, et al. Enlarged perivascular spaces in the centrum semiovale are associated with poststroke depression: A 3-month prospective study. J Affect Disord 2018;228:166-72.
15. Kongbunkiat K, Wilson D, Kasemsap N, et al. Leukoaraiosis, intracerebral hemorrhage, and functional outcome after acute stroke thrombolysis. Neurology 2017;88:638-45.

16. Charidimou A, Shoamanesh A, Wilson D, et al. Cerebral microbleeds and postthrombolysis intracerebral hemorrhage risk Updated meta-analysis. Neurology 2015;85:927-4.

17. Wei CC, Zhang ST, Wang YH, et al. Association between leukoaraiosis and hemorrhagic transformation after cardioembolic stroke due to atrial fibrillation and/or rheumatic heart disease. J Neurol Sci 2017;378:94-9.

18. Jickling GC, Liu D, Stamova B, et al. Hemorrhagic transformation after ischemic stroke in animals and humans. J Cereb Blood Flow Metab 2014;34:185-99.

19. Liu J, Zheng L, Cheng Y, et al. Trends in Outcomes of Patients With Ischemic Stroke Treated Between 2002 and 2016: Insights From a Chinese Cohort. Circ Cardiovasc Qual Outcomes 2019;12:e005610.

20. Aho K, Harmsen P, Hatano S, et al. Cerebrovascular disease in the community: results of a $\mathrm{WHO}$ collaborative study. Bull World Health Organ 1980;58:113-30.

21. Fazekas F, Chawluk JB, Alavi A, et al. MR signal abnormalities at $1.5 \mathrm{~T}$ in Alzheimer's dementia and normal aging. AJR Am J Roentgenol 1987;149:351-6.

22. Klarenbeek $P$, van Oostenbrugge RJ, Rouhl RP, et al. Ambulatory blood pressure in patients with lacunar stroke: association with total MRI burden of cerebral small vessel disease. Stroke 2013;44:2995-9.

23. Staals J, Makin SD, Doubal FN, et al. Stroke subtype, vascular risk factors, and total MRI brain small-vessel disease burden. Neurology 2014;83:1228-34.

24. Ott BR, Zamani A, Kleefield J, et al. The clinical spectrum of hemorrhagic infarction. Stroke 1986;17:630-7.

25. Hacke W, Kaste M, Bluhmki E, et al. Thrombolysis with alteplase 3 to 4.5 hours after acute ischemic stroke. $\mathrm{N}$ Engl J Med 2008;359:1317-29.

26. National Institute of Neurological Disorders and Stroke rt-PA Stroke Study Group. Tissue plasminogen activator for acute ischemic stroke. N Engl J Med 1995;333:1581-7.

27. Francis F, Ballerini L, Wardlaw JM. Perivascular spaces and their associations with risk factors, clinical disorders and neuroimaging features: A systematic review and metaanalysis. Int J Stroke 2019;14:359-71.

28. Rouhl RP, van Oostenbrugge RJ, Knottnerus IL, et al. Virchow-Robin spaces relate to cerebral small vessel disease severity. J Neurol 2008;255:692-6.

29. Martinez-Ramirez S, Pontes-Neto OM, Dumas AP, et al. 
Topography of dilated perivascular spaces in subjects from a memory clinic cohort. Neurology 2013;80:1551-6.

30. Hurford R, Charidimou A, Fox Z, et al. MRI-visible perivascular spaces: relationship to cognition and small vessel disease MRI markers in ischaemic stroke and TIA. J Neurol Neurosurg Psychiatry 2014;85:522-5.

31. Doubal FN, MacLullich AM, Ferguson KJ, et al. Enlarged perivascular spaces on MRI are a feature of cerebral small vessel disease. Stroke 2010;41:450-4.

32. Charidimou A, Meegahage R, Fox Z, et al. Enlarged perivascular spaces as a marker of underlying arteriopathy in intracerebral haemorrhage: a multicentre MRI cohort study. J Neurol Neurosurg Psychiatry 2013;84:624-9.

33. Charidimou A, Boulouis G, Pasi M, et al. MRI-visible perivascular spaces in cerebral amyloid angiopathy and

Cite this article as: Song Q, Cheng Y, Wang Y, Liu J, Wei C, Liu M. Enlarged perivascular spaces and hemorrhagic transformation after acute ischemic stroke. Ann Transl Med 2021;9(14):1126. doi: 10.21037/atm-21-1276 hypertensive arteriopathy. Neurology 2017;88:1157-64.

34. Wei C, Liu J, Li J, et al. A Non-linear Association Between Total Small Vessel Disease Score and Hemorrhagic Transformation After Ischemic Stroke With Atrial Fibrillation and/or Rheumatic Heart Disease. Front Neurol 2019;10:769.

35. Best JG, Barbato C, Ambler G, et al. Association of enlarged perivascular spaces and anticoagulant-related intracranial hemorrhage. Neurology 2020;95:e2192-9.

36. Wardlaw JM, Smith C, Dichgans M. Mechanisms of sporadic cerebral small vessel disease: insights from neuroimaging. Lancet Neurol 2013;12:483-97.

37. Li S, Hafeez A, Noorulla F, et al. Preconditioning in neuroprotection: From hypoxia to ischemia. Prog Neurobiol 2017;157:79-91. 


\section{Supplementary}

Table S1 Spearman's rank correlation coefficient among EPVS, lacunes, and WMH

\begin{tabular}{lcc}
\hline & BG-EPVS & CS-EPVS \\
\hline BG-EPVS & - & $0.243^{*}$ \\
Lacunes & $0.237^{*}$ & 0.035 \\
CWMH & $0.526^{*}$ & 0.065 \\
PWMH & $0.515^{*}$ & 0.024 \\
DWMH & $0.450^{*}$ & 0.086 \\
\hline
\end{tabular}

BG-EPVS: basal ganglia enlarged perivascular spaces; CS-EPVS: centrum semiovale enlarged perivascular spaces; cWMH: total cerebral white matter hyperintensity; PWMH: periventricular white matter hyperintensity; $\mathrm{DWMH}$ : deep white matter hyperintensity. ${ }^{*} \mathrm{P}<0.001$.

Table S2 Imaging characteristics of patients with and without PH

\begin{tabular}{|c|c|c|c|}
\hline Imaging characteristics & Without $\mathrm{PHN}=456$ & With $\mathrm{PHN}=38$ & $P$ \\
\hline Total EPVS, median (IQR) & $4(3-5)$ & $4(3-5)$ & 0.407 \\
\hline BG-EPVS, median (IQR) & $1(1-2)$ & $1(1-2)$ & 0.660 \\
\hline Moderate to severe BG-EPVS, $n$ (\%) & $223(48.9)$ & $16(42.1)$ & 0.420 \\
\hline CS-EPVS, median (IQR) & $2(2-3)$ & $2(2-3)$ & 0.324 \\
\hline Moderate to severe CS-EPVS, n (\%) & $401(87.9)$ & $34(89.5)$ & 0.984 \\
\hline Lacunes, n (\%) & 89 (19.5) & $6(15.8)$ & 0.575 \\
\hline PWMH, median (IQR) & $1(0-2)$ & $1(0-1)$ & 0.583 \\
\hline Extensive PWMH, n (\%) & $38(8.3)$ & $1(2.6)$ & 0.348 \\
\hline DWMH, median (IQR) & $1(0-1)$ & $1(0-1)$ & 0.443 \\
\hline Extensive DWMH, n (\%) & $84(18.4)$ & $6(15.8)$ & 0.686 \\
\hline cWMH, median (IQR) & & & 0.354 \\
\hline No cWMH, n (\%) & $119(26.1)$ & $9(23.7)$ & \\
\hline Mild cWMH, n (\%) & $206(45.2)$ & $22(57.9)$ & \\
\hline Moderate cWMH, n (\%) & $90(19.7)$ & $6(15.8)$ & \\
\hline Severe cWMH, n (\%) & $41(9.0)$ & $1(2.6)$ & \\
\hline Total CSVD burden, median (IQR) & $1(0-1)$ & $0(0-1)$ & 0.298 \\
\hline Score 0, n (\%) & $199(43.6)$ & $20(52.6)$ & 0.627 \\
\hline Score 1, n (\%) & $146(32.0)$ & $11(28.9)$ & \\
\hline Score 2, n (\%) & 79 (17.3) & $4(10.5)$ & \\
\hline Score 3, n (\%) & $32(7.0)$ & $3(7.9)$ & \\
\hline
\end{tabular}

PH: parenchymal hematoma; BG-EPVS: basal ganglia enlarged perivascular spaces; CS-EPVS: centrum semiovale enlarged perivascular spaces; PWMH: periventricular white matter hyperintensity; DWMH: deep white matter hyperintensity; cWMH: total cerebral white matter hyperintensity; CSVD: cerebral small vessel disease; IQR: interquartile range.

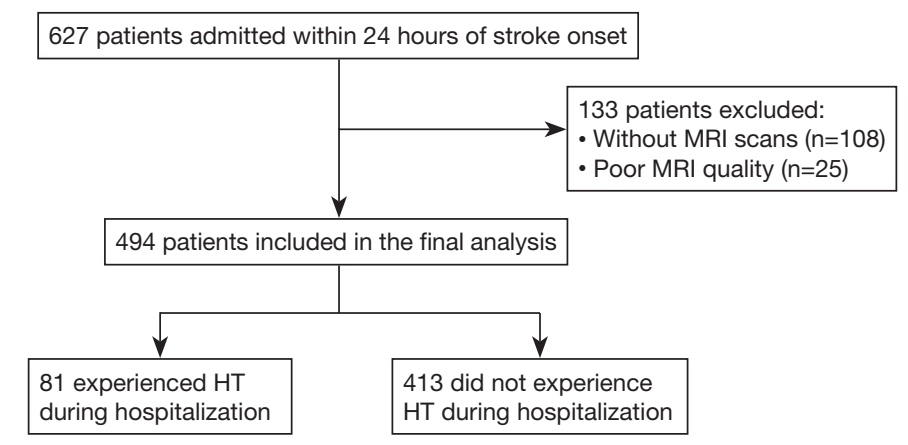

Figure S1 Flowchart of patients' enrollment. 